Rabaska

Revue d'ethnologie de l'Amérique française

\title{
Chaire de recherche du Canada en oralité des francophonies minoritaires et Centre acadien (Université Sainte-Anne)
}

\section{Jean-Pierre Pichette}

Volume 4, 2006

URI : https://id.erudit.org/iderudit/201797ar

DOI : https://doi.org/10.7202/201797ar

Aller au sommaire du numéro

Éditeur(s)

Société québécoise d'ethnologie

ISSN

1703-7433 (imprimé)

1916-7350 (numérique)

Découvrir la revue

Citer ce document

Pichette, J.-P. (2006). Chaire de recherche du Canada en oralité des

francophonies minoritaires et Centre acadien (Université Sainte-Anne).

Rabaska, 4, 214-218. https://doi.org/10.7202/201797ar 


\section{Rapports des institutions}

ACADIE

\section{Chaire de recherche du Canada en oralité des francophonies minoritaires}

CoFram / Centre acadien

Téléphone : (902) 769-2114

Université Sainte-Anne

Télécopieur : (902) 769-0063

Pointe-de-l'Église

(Nouvelle-Écosse) B0W 1M0

Courriel : jeanpierre.pichette@usainteanne.ca

Toile : www.usainteanne.ca

Parmi les faits saillants de cette deuxième année de la chaire Cofram, l'inauguration du Laboratoire de littérature orale (LABOR) en octobre 2005 et le versement au Centre acadien $(\mathrm{CA})$ de la collection du titulaire sont à signaler, comme aussi la synergie qui se développe autour de la chaire par des partenariats dans le secteur de l'enseignement et de la recherche.

\section{Enseignement}

Dans le cadre de ses tâches pédagogiques, le titulaire de la Cofram a collaboré à la restauration du baccalauréat en Études acadiennes de l'Université SainteAnne. Soutenu par un comité d'orientation, composé de collègues du département d'Études françaises et des Sciences humaines, il pilota la proposition qui reçut la sanction des divers comités de l'université pendant l'automne 2005 et fut ratifiée par la Commission des études supérieures des provinces maritimes (CÉSPM) en avril 2006. Ce programme, qui inclut un bloc de onze cours d'ethnologie (dont deux transcodés en Études françaises), commencera dès l'automne prochain.

\section{Recherche}

Pareillement, la CoFram a servi d'ancrage au regroupement d'une quinzaine de professeurs qui, à la fin d'août 2005, ont convenu de former le Groupe de recherche en études acadiennes (GRÉA) de l'Université Sainte-Anne. Déjà engagés dans des activités scientifiques, ces chercheurs comptent que le GRÉA sera un lieu de partage et d'entraide mutuelle en vue de l'avancement des connaissances en ce domaine ; ils s'alignent ainsi sur la mission de l'Université Sainte-Anne, qui vise au développement de la communauté acadienne et à la 
promotion de sa culture, par la mise en valeur de l'histoire, de la littérature, de la langue, des traditions et des mours des Acadiens. Ce collectif publie un bulletin annuel d'information (GRÉA, $\mathrm{n}^{\circ}$ 1, automne 2005, Pointe-de-l'Église, Université Sainte-Anne, $4+2$ p. ; disponible au site www.usainteanne.ca/ documents/GREA-1.pdf)) et, comme on le verra ci-dessous, il collabore activement aux travaux de la COFRAM.

\section{Réunions scientifiques}

A. JOURNÉES D'ÉTUDES - Mettant en œuvre une partie essentielle de son mandat - soit encourager les recherches et les études comparées sur la littérature orale et les traditions populaires des minorités acadiennes et canadiennesfrançaises d'Amérique du Nord -, la chaire CoFram a tenu ses premières journées d'études à la chapelle et au CA de l'Université Sainte-Anne les 25 et 26 octobre 2005. À cette occasion, un petit noyau composé de spécialistes européens, de France et de Belgique, et de leurs collègues de l'Acadie, du Québec et d'autres régions de la francophonie nord-américaine partagèrent leurs réflexions sur la notion de Résistance des marges afin de circonscrire les grands axes d'un colloque international prévu pour l'été 2007. Organisée en partenariat avec le Groupe de recherche en études acadiennes de l'Université Sainte-Anne (GRÉA), le Centre de recherche bretonne et celtique de l'Université de Bretagne occidentale ( $\mathrm{CRBC}_{\mathrm{RB}}$, Brest) et la Société québécoise d'ethnologie (SQE), cette réunion fut suivie à Brest, les 5 et 6 juin 2006, par la création des Rencontres britto-franco-canadiennes autour du thème Traditions, revitalisations, créations, cette fois sous la responsabilité du CRBC, notre partenaire français.

B. COLLOQUe - Toujours en étroite collaboration avec le GréA, la COFRAM a organisé un colloque national : le Patrimoine religieux de la Nouvelle-Écosse. Signes et paradoxes en Acadie. L'événement, qui eut lieu à la salle MarcLescarbot de l'Université Sainte-Anne, a rassemblé du 18 au 21 juin 2006, plus de 80 participants. Les conférenciers ont examiné certaines des traces qu'imprime le patrimoine religieux dans l'univers culturel des Acadiens de la Nouvelle-Écosse : signes matériels ou visibles - églises, cimetières et autres monuments - et signes spirituels relevant de l'institution officielle ou de la réinterprétation populaire - rites et pratiques, et les liens très étroits qui unissent le culte à la culture. Ces délibérations, éclairées par l'expérience du Québec et de l'Ontario français, entendaient faire le point sur la situation du patrimoine religieux de la Nouvelle-Écosse et sensibiliser la population acadienne à cette portion significative de sa culture, aux dangers qui la menacent en explorant les voies qui lui garantiront un avenir. On compte 
réunir les communications de la vingtaine de conférenciers invités dans des actes de colloque à paraître prochainement. Le personnel du Centre acadien et les étudiants du laboratoire de littérature orale (LABOR) ont assuré au plan technique le succès de cette manifestation.

C. Chercheurs invités - En outre, cette année, la Cofram a accueilli ses premiers chercheurs invités. Du 17 au 27 octobre 2005, M. Jean Simard, président de la Société québécoise d'ethnologie (SQE) et secrétaire de la revue Rabaska, a mené une recherche exploratoire sur le patrimoine religieux de la baie Sainte-Marie, en Nouvelle-Écosse. Cette première incursion devait nourrir ses exposés à nos journées d'études des jours suivants sur la résistance des marges et au colloque de juin dernier sur le patrimoine religieux acadien. Du 6 au 9 février 2006, c'est M. Yves Frenette, historien et directeur du département d'Études pluridisciplinaires du Collège Glendon (Université York), qui fut l'invité de la chaire pour la deuxième des conférences de la COFRAM. Le 7 février 2006, il entretint son auditoire sur « La lettre dans la diaspora francophone d'Amérique, $\mathrm{XIX}^{\mathrm{e}}-\mathrm{XX}^{\mathrm{e}}$ siècles ». Son exposé, fondé sur la recherche qu'il dirige avec Marcel Martel et John Willis, s'appuyait sur les excellentes collections du Centre acadien, notamment la riche correspondance d'une famille qui compte 1176 lettres et s'étend sur une centaine d'années.

\section{Laboratoire de littérature orale}

A. INAUGURATION - À la clôture des journées d'études, le 25 octobre 2005, on inaugura la salle de travail aménagée pour le LABOR au Centre acadien. Les invités ont pu voir les postes de travail prêts à accueillir les étudiants et les chercheurs engagés dans les projets de la chaire ; ils ont aussi appris que le $\mathrm{CA}_{\mathrm{A}}$ avait acquis l'outillage technique nécessaire pour enregistrer, traiter et conserver les documents rassemblés par l'enquête orale. Financé par la Fondation canadienne pour l'innovation, le matériel du laboratoire a été choisi dans le but de permettre les activités alternées de deux équipes qui pourront se relayer sur le terrain et en cabinet de travail.

B. Dépôts - Puis, désirant marquer de façon toute spéciale sa promesse d'enrichissement documentaire, le titulaire versa officiellement au laboratoire la collection intégrale des 3750 premiers enregistrements sonores qu'il a réunis au cours des ans à la faveur de ses missions ethnologiques dans neuf provinces canadiennes, dont trois acadiennes ; pour consulter les quelque 200 heures de sa collection, disponible sur disques compacts, il déposa en outre l'outil de recherche indispensable pour y accéder, soit l'inventaire 
récemment informatisé. Aussi, à la reprise des occupations estivales du LABOR, une équipe de cinq étudiants a-t-elle pu continuer la saisie de la collection orale de J.-P. Pichette, parachever la transcription préliminaire de la documentation recueillie en Acadie et même avancer le traitement de celle du Québec, de l'Ontario et des provinces de l'Ouest. Pour sa part, le technicien a numérisé les enregistrements vidéo de cette collection (environ 30 heures). Ces travaux prioritaires fondent le projet d'édition d'un corpus de littérature orale (ÉCLORE), l'un des volets constitutifs de la chaire. Au cours de l'année qui s'achève, le LABOR aura versé dans les collections du Centre acadien une soixantaine d'heures de nouveaux enregistrements, soit les disques audio du terrain de l'été 2005 , et les disques vidéo des journées d'études 2005 et du colloque 2006 .

C. GUIDE D'ÉCOUTE - Pour sa part, un membre de l'équipe a entrepris le travail d'indexation des autres collections orales du Centre acadien enregistrées entre 1972 et 1978 ; la préparation du guide d'écoute qu'il prépare devrait faciliter le repérage parmi les 330 heures d'entrevues que compte cette documentation.

D. TERRAIN - Ce volet de travail en cabinet du LABOR est nécessairement assorti d'un volet terrain. Cet été, l'équipe a mené une deuxième mission de terrain, élargissant par un nouveau projet pilote la cueillette à une demidouzaine de thèmes relevant des traditions orales et des célébrations de la vie privée et des cycles calendaires, toujours auprès de la population acadienne de la Nouvelle-Écosse.

\section{Publications}

A. RABASKA - Grâce à son équipe permanente, secrétaire et technicien, et à l'équipe des étudiants du LABOR, le titulaire a pu poursuivre ses activités ordinaires. Parmi celles-ci, la coordination de la revue Rabaska n'est pas la moindre. C'est à partir de la CofRAM que cette revue, la seule qui se voue exclusivement à l'étude des traditions populaires de l'Amérique française, est produite. Le troisième volume a été publié à l'automne 2005 (Québec, SQE, vol. 3, 2005, 250 p.) et le quatrième est en voie d'achèvement. Comme c'est l'habitude, chaque volume compte une cinquantaine d'auteurs et une trentaine d'autres collaborateurs.

B. Autres - En plus d'assurer la rédaction des Cahiers Charlevoix, dont le septième paraîtra en 2006, le titulaire, qui est membre de la Société Charlevoix, a collaboré à la brochure de ce collectif parue à l'automne 2005: Les Régionalismes de l'Ontario français. Actes de la table ronde de la Société 
Charlevoix $\left[10^{\circ}\right]$ Salon du livre de Toronto (Toronto, Éditions du GREF, 2005, [6]-81 p. Il a également participé au beau livre Les Monuments intellectuels québécois du XX' siècle sous la direction de Claude Corbo (Septentrion, 2006) dans lequel il publie le texte consacré au livre de Marius Barbeau. Enfin, la chaire s'est aussi dotée d'un bulletin annuel de nouvelles dont le premier numéro paraissait l'automne dernier : La Mouvée, bulletin d'information de la Cofram, $\mathrm{n}^{\circ} 1$, décembre $2005,8 \mathrm{p}$.; il est disponible au site www.usainteanne.ca/documents/la_mouvee_1.pdf.

Jean-Pierre Pichette 